Reprod. Nutr. Dévelop., 1987, 27 (3), 701-705.

\title{
Mesure du taux d'ovulation par cœlioscopie chez la lapine
}

\author{
Michèle THEAU-CLEMENT, G. BOLET
}

Station d'Amélioration génétique des Animaux, I.N.R.A. B.P. 27, 31326 Castanet Tolosan Cedex, France.

Summary. Coelioscopic measurement of ovulation rate in doe rabbits.

With the aim of determining the efficiency of a simple and non destructive method for measuring the ovulation rate, 20 doe rabbits were subjected to coelioscopy and slaughtered on day 14 of gestation. Among the $\mathbf{4 0}$ ovaries examined, $45 \%$ of corpora lutea counts were accurate and $35 \%$ approximate $( \pm 1)$, i.e. a total of $80 \%$ acceptable counts. This percentage represented $96 \%$ when the number of corpora lutea per ovary did not exceed 8. As compared with a few control does, coelioscopy did not seem to have a significant unfavourable effect on embryonic survival rate on day 14 of gestation, but this result has to be checked. It may be concluded that this method can be used in further experiments.

\section{Introduction.}

Pour analyser la mortalité embryonnaire dans les espèces polytoques, il est nécessaire de connaître le taux d'ovulation. Chez la lapine, il est classiquement mesuré soit par abattage à un stade donné de la gestation, soit par laparotomie et observation de l'ovaire. Ces deux techniques présentent de nombreux inconvénients, alors que dans d'autres espèces comme les porcins (Locatelli, 1971), cette observation est depuis longtemps faite par cœelioscopie. Nous avons étudié l'efficacité de cette technique chez la lapine, sa fiabilité et son influence éventuelle sur la viabilité des embryons.

\section{Matériel et méthodes.}

1. Matériel animal. - 28 lapines nullipares, âgées de 4 mois environ, appartenant à la souche INRA 1066, issue de la race Californienne, ont été utilisées. 22 ont été saillies par des mâles ; 6 ont refusé l'accouplement et ont reçu une injection par voie intra-veineuse de $60 \mathrm{UI}$ d'hCG. Vingt lapines ont subi une cœlioscopie 8 jours après la saillie ou l'injection (J8) pour déterminer le nombre (NO) de corps jaunes sur chaque ovaire. Ces 20 lapines et les 8 autres servant de témoins ont été abattues à j14 ; le tractus génital a été prélevé pour compter les corps jaunes sur cha- 
que ovaire (NR), et dans chaque corne utérine les embryons vivants, morts et les sites d'implantation vides.

2. Déroulement de la cœlioscopie. - Nous avons comparé deux endoscopes de type arthroscope, de 35 et $45 \mathrm{~mm}$ de diamètre et de 12 à $15 \mathrm{~cm}$ de longueur. La lapine est anesthésiée par inhalation en circuit semi-ouvert de fluothane véhiculé par de l'oxygène, à une concentration de $5 \%$ pour induire l'anesthésie, puis de 2 à $3 \%$ pendant l'observation, avec un débit de 0,100 à $0,250 \mathrm{I} / \mathrm{min}$. Quand la myorésolution est atteinte, l'abdomen est rasé et désinfecté sur $8 \mathrm{~cm}$ en dessous de l'attache ombilicale et sur $3 \mathrm{~cm}$ de part et d'autre de la ligne médiane ; la lapine est installée sur une table d'opération inclinée de 20 à 30 degrés environ vers I'avant.

Une aiguille de Veres est introduite à $6 \mathrm{~cm}$ en dessous de l'ombilic. Elle sert à insuffler l'air et son extrémité arrondie permet les manipulations nécessaires pour mettre l'ovaire en évidence. Une incision à $3 \mathrm{~cm}$ de l'ombilic facilite l'introduction du trocart qui est ensuite retiré du fourreau et remplacé par l'endoscope relié à un générateur de lumière de 250 watts. Les corps jaunes sont comptés successivement sur chaque ovaire. L'incision est ensuite fermée par un point de suture et on fait une injection intra-musculaire de 500000 Ul d'antibiotique (Spécilline G).

3. Analyses statistiques. - Soit D la différence entre le nombre de corps jaunes comptés par endoscopie et dénombrés après abattage ( $D=N O-N R$ ). Pour ce premier essai nous avons défini comme "acceptables" les comptages exacts $(D=0$ ) et ceux approchés à \pm 1 près, et comme " inacceptables " ceux pour lesquels $D$ est supérieur ou inférieur à 1 . Le pourcentage de comptages exacts et acceptables a été calculé sur l'ensemble des données et en fonction du nombre NR de corps jaunes par ovaire réparti en 4 classes : 0 à 4,5 à 8,9 à 12 et 13 à 16 . Une analyse de variance sur $D$ avec les effets appareil (2 niveaux), opérateur ( 2 niveaux) et NR (4 niveaux) a également été réalisée.

Le taux d'ovulation, le nombre d'embryons et de sites d'implantation, le taux d'implantation et de survie des embryons à 14 jours de gestation des 16 lapines expérimentales et des 6 lapines témoins ayant accepté la saillie ont été comparés par des tests de Student.

\section{Résultats}

\section{Précision du comptage.}

Sur l'ensemble des 40 ovaires examinés, l'erreur $D$ est égale à $-0,45$ $\pm 0,26.45 \%$ des comptages sont exacts et $80 \%$ sont acceptables (fig. 1). Les comptages faux le sont plus souvent par défaut $(15 / 22)$ que par excès.

L'analyse de variance ne met pas en évidence d'effets significatifs de l'endoscope, de l'opérateur ou du nombre de corps jaunes (tabl. 1). La figure 2 montre toutefois que le pourcentage de comptages acceptables diminue quand le taux d'ovulation augmente. Quand le nombre de corps jaunes par ováire ne dépasse pas $8,96 \%$ des comptages (26/27) sont acceptables, alors que seulement $46 \%$ $(6 / 13)$ sont acceptables pour plus de 8 corps jaunes $\left(\chi^{2}=13,8 \mathrm{P}>0,01\right)$. 


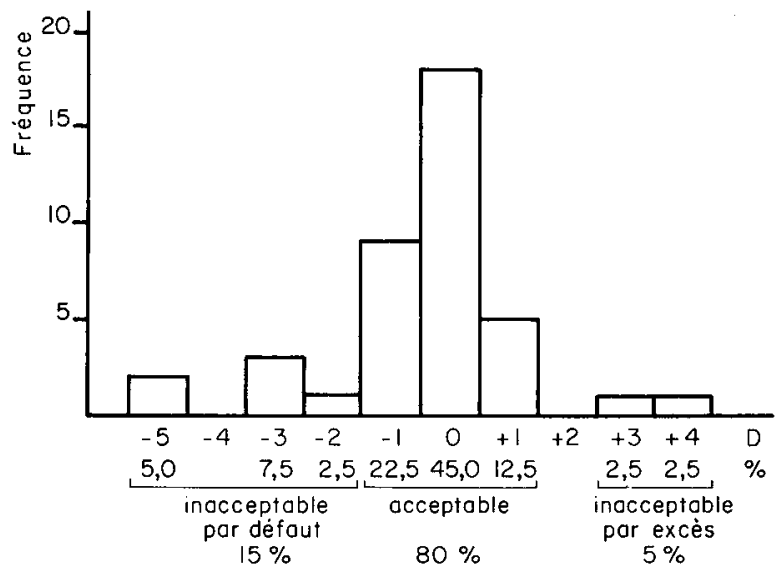

FIG. 1. - Distribution de la précision du comptage par ovaire.

$\mathrm{D}=$ Nombre de corps jaunes comptés par endoscopie - nombre de corps jaunes comptés à l'abattage.

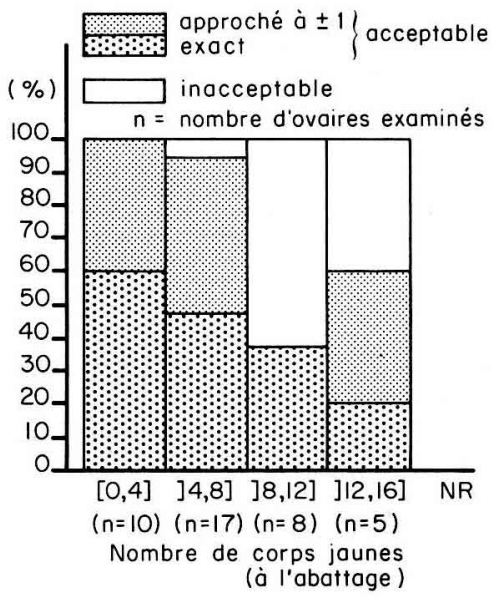

FIG. 2. - Fréquence de comptages exacts $(\mathrm{D}=0)$ et acceptables $(-1 \leq \mathrm{D} \leq 1)$ en fonction du nombre de corps jaunes par ovaire (à l'abattage).

2. Effets de l'endoscopie sur la survie embryonnaire.

Sur les 22 femelles saillies, il n'apparaît pas de différence nette du taux de gestation entre les 16 femelles expérimentales et les 6 témoins, puisque, dans chaque groupe, 2 ne sont pas gestantes. Les tests $t$ ne mettent pas en évidence de différence entre les 2 groupes de femelles gestantes pour le nombre d'embryons totaux et vivants par corne, ni pour le taux de survie des embryons à 14 jours (tabl. 2). Il apparaît par contre une différence significative, en rejetant I'hypothèse d'égalité des variances, pour le nombre d'embryons morts + sites 
vides $(+0,69$ chez les femelles expérimentales). En fait, cette différence est due essentiellement à la présence de 5 embryons morts chez 2 lapines expérimentales. On ne peut donc pas exclure la possibilité d'un risque de mortalité des embryons accru chez les lapines soumises à l'endoscopie.

TABLEAU 1

Résultats de l'analyse de variance des effets endoscope, operateur et taux d'ovulation par ovaire sur $D$.

\begin{tabular}{|c|c|c|c|}
\hline Effet & d.d.I. & $F$ & diff. \\
\hline 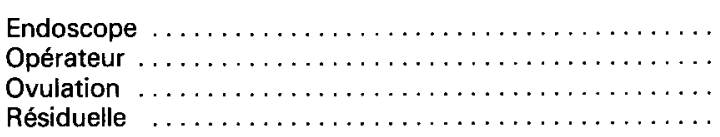 & $\begin{array}{r}1 \\
1 \\
3 \\
34\end{array}$ & $\begin{array}{l}1,5 \text { n.s. } \\
2,3 \text { n.s. } \\
0,4 \text { n.s. } \\
\left(\tau_{\mathrm{e}}^{2}=2,72\right)\end{array}$ & $\begin{array}{l}0,71 \pm 0,58 \\
0,82 \pm 0,55\end{array}$ \\
\hline
\end{tabular}

d.d.I. : Degrés de liberté ; F : F de Fisher ; n.s. : non significatif ; diff. : Différence \pm écart-type entre les 2 niveaux de l'effet; $\tau_{\mathrm{B}}^{2}$ : Variance résiduelle.

TABLEAU 2

Comparaison de la survie embryonnaire chez les lapines soumises à l'endoscopie et chez les lapines témoins.

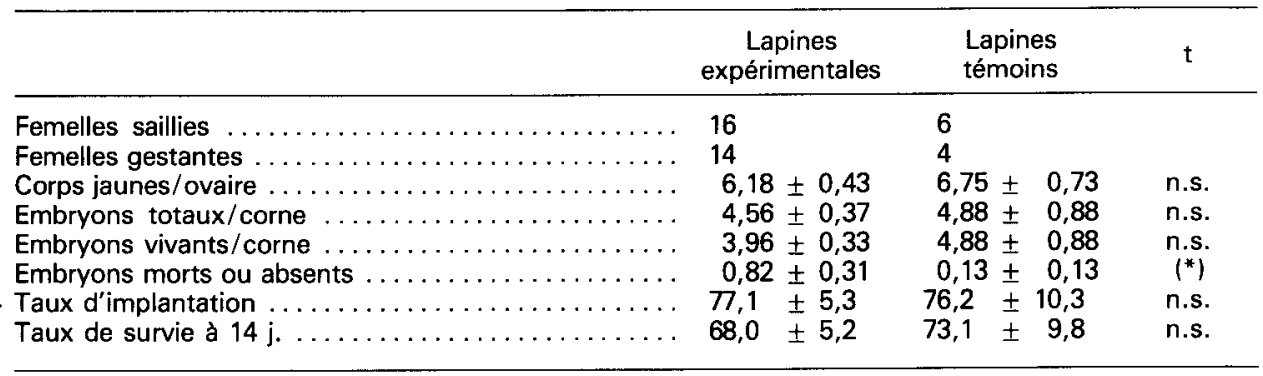

$\mathbf{t}$ : test de Student de la différence entre lots ; n.s. : non significatif ; $\left(^{*}\right) \mathbf{P}<0,05$ (test approché en raison de l'inégalité des variances).

\section{Discussion.}

Si nous comparons ces premiers résultats à ceux obtenus par Locatelli (1971) lors de ses premiers essais sur truies ( $27 \%$ de dénombrements exacts et $70 \%$ d'acceptables par truie), nous pouvons considérer que ce premier essai est encourageant. Bien que l'analyse de variance ne mette en évidence aucun effet significatif, l'erreur moyenne varie avec l'appareil et surtout avec l'opérateur (tabl. 1) ; le choix du meilleur endoscope et l'entraînement des opérateurs ne peuvent qu'améliorer les résultats.

Par ailleurs, la figure 2 met en évidence une influence du taux d'ovulation sur la précision du comptage. II faut toutefois signaler que les femelles ayant reçu une 
injection d'hCG ont eu un taux d'ovulation exceptionnellement élevé 113,7 corps jaunes par ovaire). Sur les 27 comptages correspondant à un nombre de corps jaunes par ovaire inférieur ou égal à 8 , ce qui correspond au cas le plus courant dans nos souches (Hulot et Matheron, 1979), le taux de comptages acceptables (96\%) est par contre tout à fait satisfaisant. L'erreur la plus fréquente est une sous-estimation d'une unité du nombre de corps jaunes ; elle représente 22,5\% des cas (fig. 1). En effet, il est difficile d'observer les corps jaunes à l'extrémité de l'ovaire, souvent cachée par le pavillon. Dans la mesure où les lapines que nous observons sont des futures reproductrices, il sera bon de vérifier si une recherche plus prolongée, à l'aide du manipulateur, des corps jaunes sous le pavillon, ne risque pas de provoquer des lésions. Nos données ne semblent pas mettre en évidence d'effet défavorable de l'endoscopie sur le taux de survie des embryons. L'accroissement du nombre d'embryons morts demande toutefois à être vérifié en distinguant, si nécessaire, les effets respectifs de l'anesthésie et de la manipulation.

\section{Conclusion.}

Nous avons montré que l'examen des ovaires par cœlioscopie est praticable chez la lapine et peut donc remplacer avantageusement l'abattage ou la laparotomie. La fiabilité de cette méthode, d'ores et déjà encourageante, devrait encore augmenter avec l'entraînement. II reste néanmoins à vérifier sur un effectif plus grand que la cœlioscopie n'a pas d'effet sur la survie embryonnaire.

Reçu en janvier 1987.

Accepté en février 1987.

Remerciements. - Nous tenons à remercier A. Locatelli et D. Lajous pour nous avoir initiés à cette technique, les sociétés Pouret et Wolf pour nous avoir prêté du matériel. Cette expérience a été réalisée dans le cadre de l'AIP-INRA, $n^{\circ} 4528$.

\section{Références}

HULOT F., MATHERON G., 1979. Analyse des variations génétiques entre trois races de lapins de la taille de portée et de ses composantes biologiques en saillie post-partum. Ann. Génét. Sél. anim., 11, 53-77.

LOCATELLI A., 1971. Technique d'examen cœlioscopique des ovaires de la truie. Ann. Biol, anim. Bioch. Biophys., 11, 495-498. 